\title{
Whole Brain CT Perfusion after Cerebral Air Embolism
}

\author{
Amanda Murphy, Carlos Torres, Cheemun Lum, Mathew Hogan, Miguel Bussière
}

Can. J. Neurol. Sci. 2011; 38: 522-525

Cerebral air embolism (CAE) occurs as a result of air entering the arterial or venous circulation. Iatrogenic causes of CAE are found across all specialties, and include inadvertent air entry during cardiac catheterization, angiography, lung biopsy or via an intravascular catheter ${ }^{1,2}$. Ischemic brain injury occurs when air emboli directly obstruct intracranial arteries or cause inflammation, blood brain barrier breakdown and cerebral edema which secondarily interfere with blood supply to the brain $^{1,2}$.

Patients with CAE develop sudden neurological symptoms which vary based on the absolute volume of air introduced and extent of brain affected. Symptoms are non-specific and range from confusion or disorientation to coma, seizures or focal deficits such as hemiparesis ${ }^{1}$. A high index of suspicion is required when neurological symptoms develop in temporal association with surgical or invasive procedures that carry a risk of air embolism ${ }^{3}$. Consideration of a diagnosis of CAE may be delayed or masked in patients that have received general anesthesia or conscious sedation ${ }^{1}$.

Neuro-imaging can be helpful in establishing a diagnosis of CAE. Air emboli may be directly demonstrated by non-enhanced computed tomography (NECT), however, microscopic CAE or small volumes of intravascular air may be missed. Air is rapidly absorbed from the cerebral circulation and thus delays in acquiring brain imaging may result in false negative computed tomography (CT) scans ${ }^{4}$. The use of magnetic resonance imaging (MRI) is limited by the time delay in obtaining imaging and lengthy scan times both of which are undesirable in potentially unstable patients. A rapid imaging method that can accurately demonstrate CAE would thus be of use.

We report two cases of cerebral air embolism evaluated acutely using an imaging protocol consisting of NECT of the head, computed tomogram angiography (CTA) of the head and neck and whole brain CT perfusion (CTP). To the best of our knowledge, this is the first report of CTP use in the acute evaluation of suspected CAE.

\section{Case Report \#1}

A patient with non-small cell lung carcinoma underwent diagnostic bronchoscopy with transbronchial biopsies. Minor bleeding was noted during the procedure, and therefore epinephrine was injected at the biopsy site. Shortly thereafter, the patient suddenly developed extensor posturing of the upper and lower extremities which lasted five minutes and then became periodic in nature. The patient was unresponsive with a normal pupillary response to light. Systolic blood pressure was elevated at $190 \mathrm{mmHg}$, there was no tachycardia and oxygen saturation was normal. Naloxone was administered to reverse the effects of fentanyl used for sedation. Flumazenil was not administered to reverse the effects of midazolam due to uncertainty as to whether the patient had suffered a seizure. The patient was intubated and mechanically ventilated for airway protection.

A CT scan of the head was obtained within one hour of symptom onset to rule out stroke or intracranial hemorrhage. The study was performed on a Toshiba Aquilion ONEC 320-slice CT scanner and included a NECT head, CTA of the head and neck and whole brain CTP. The NECT showed loss of grey-white differentiation and effacement of the overlying cerebral sulci consistent with either cerebral edema or early ischemic changes in the right cerebral hemisphere and in the left frontal lobe (Figure 1). In addition, four small round hypodensities (0 to -48 Hounsfield Units (HU) were noted in the subarachnoid space at the vertex overlying both cerebral hemispheres (Figure 1). On the CTA, the cervical carotid arteries and intracranial vessels were patent without evidence of intravascular air, thrombus or stenosis. The CTP demonstrated strikingly prolonged time to peak (TTP) and mean transit time (MTT) in both the right and left cerebral hemispheres (Figure 2, colour image available online). Cerebral blood flow (CBF) was moderately decreased in the corresponding regions with no obvious alteration in Cerebral blood volume (CBV).

Due to the clinical suspicion of air embolism and the imaging findings suggestive of intracranial air with associated ischemia, hyperbaric oxygen therapy was promptly initiated. The patient's neurological status remained unchanged throughout treatment: pupils were symmetric and reactive to light, painful stimuli elicited only extensor posturing and respiratory effort was inconsistent. Despite prompt hyperbaric oxygen therapy, diffusion weighted MRI at 48 hours post event demonstrated extensive restricted diffusion in both cerebral hemispheres consistent with ischemic infarcts (Figure 3). The patient's condition deteriorated and she expired.

From the Department of Medicine (AM, MH, MB), Department of Radiology (CT, CL), Division of Neuroradiology, Division of Neurology (MH, MB), The Ottawa Hospital,

Ottawa, Canada.

Received October 6, 2010. Final Revisions Submitted December 7, 2010.

Correspondence to: Amanda Murphy, 211 - 380 Macpherson Avenue, Toronto, Ontario, M4V 3E3, Canada. 


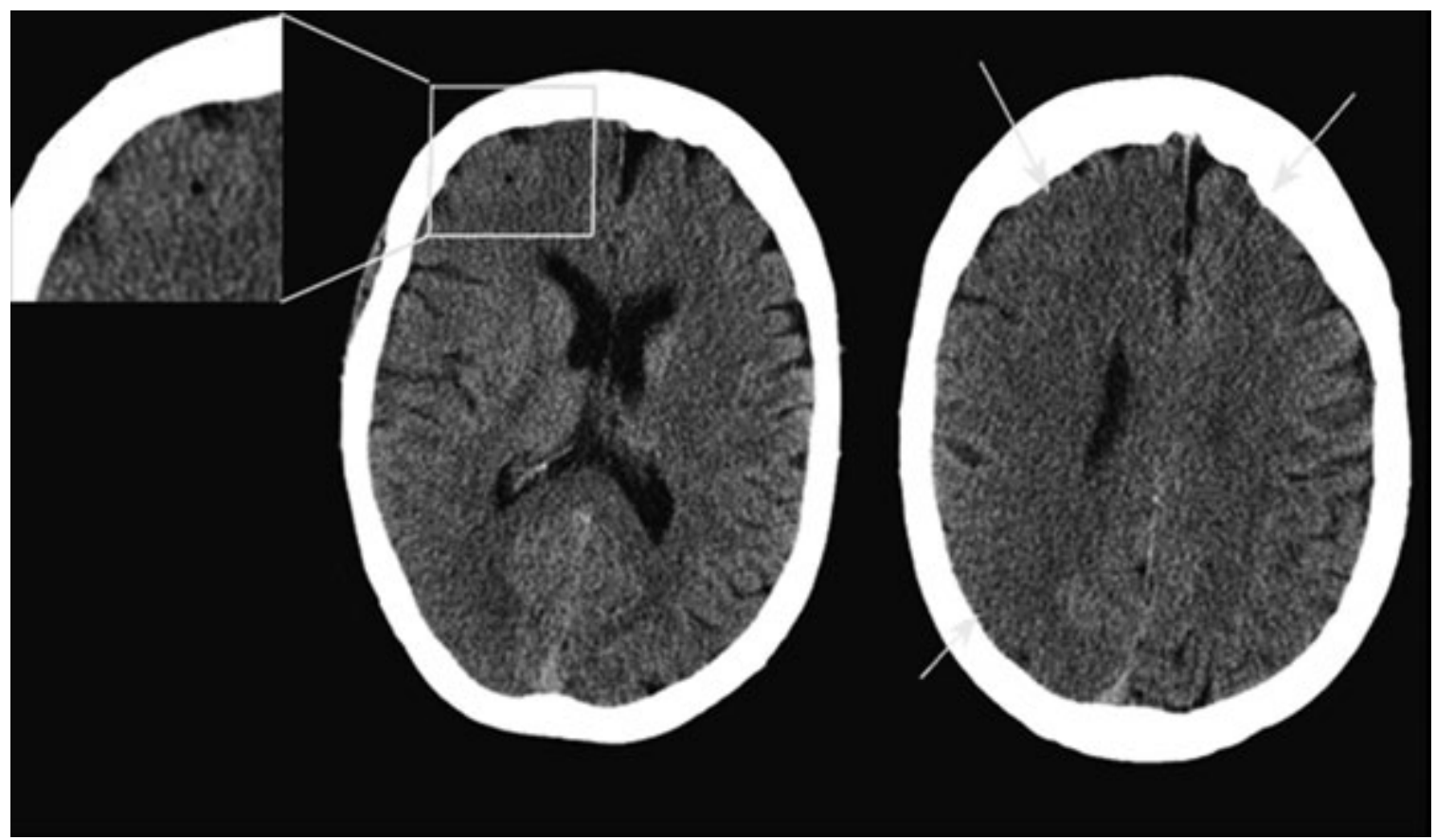

Figure 1: Case \#1 NECT shows subtle loss of grey-white differentiation and effacement of the overlying cerebral cortex consistent with edema or early ischemic change in the right cerebral hemisphere and left frontal lobe. Four small round hypodense foci were seen in the subarachnoid space in the right frontal region, in the left superior frontal region, adjacent to the superior sagittal sinus and in the right temporal-occipital region ( 0 to -48 HU).
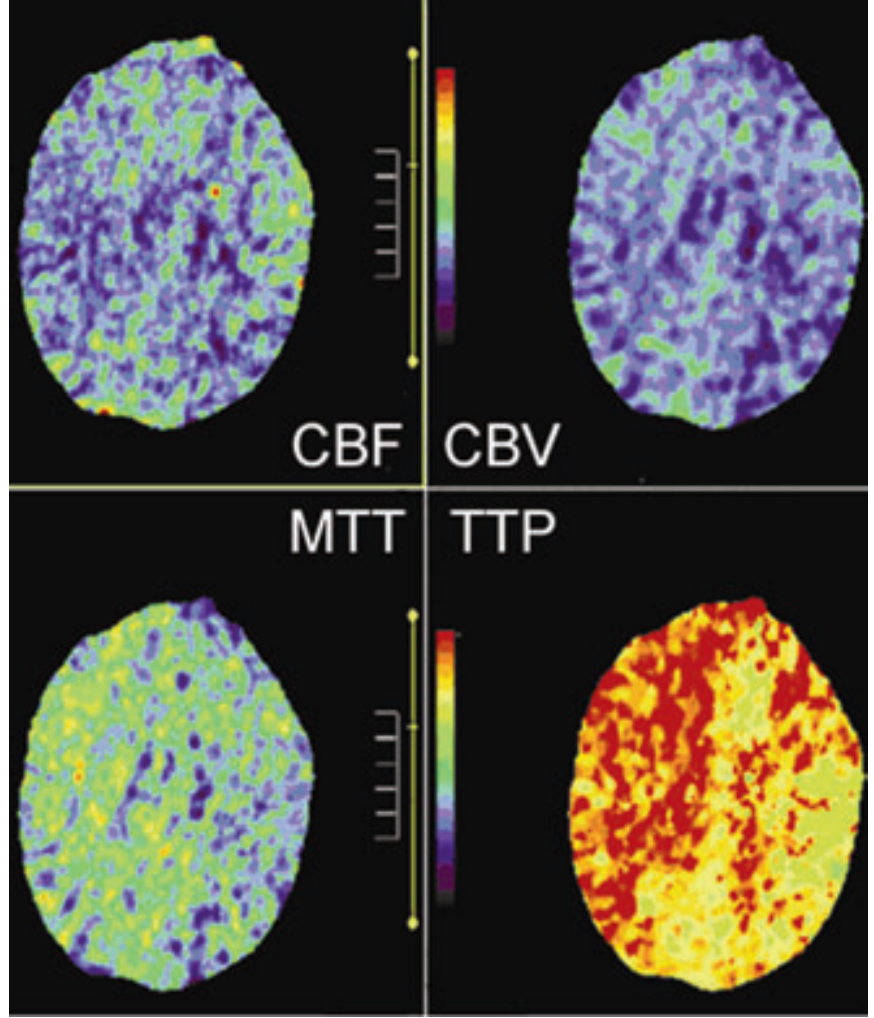

Figure 2: Case \#1 Whole brain CT perfusion. Prolonged mean-transit time (MTT) and time-to-peak (TTP) is evident in the majority of the right hemisphere, as well as in the left frontal and parietal lobes. Slight reduction in cerebral blood flow is also evident in these locations.

\section{CAse Report \#2}

An elderly patient with a history of colorectal cancer suddenly developed slurred speech and left-sided weakness. On initial assessment in the emergency room, the patient could obey some simple commands but had no verbal output. A homonymous left hemi-visual field deficit was suspected and the patient was found to have left-sided hemiplegia.

A NECT head, CTA of the head and neck and whole brain CTP were obtained within 75 minutes of symptom onset. No filling defects or significant stenoses were seen in the extra- or intra-cranial vessels. Multiple serpiginous areas of low attenuation (HU -119 and lower) were noted in the subarachnoid spaces overlying the right parietal and occipital lobes and in the right cavernous sinus (Figure 4). Subtle hypodensity was present in the subcortical white matter of the right parietal and occipital lobes. Brain CTP demonstrated a large area of decreased CBF, CBV and MTT in the corresponding right parietal and occipital region, that may represent infarction (Figure 5). A surrounding larger area of increased MTT and TTP was noted in the right frontal and temporal lobes in keeping with ischemic penumbra.

Imaging findings were consistent with cerebral air embolism. Due to the patient's poor functional status, long-term prognosis and prior expressed wishes, hyperbaric oxygen therapy was not pursued and the patient subsequently passed away.

\section{DISCUSSION}

Arterial embolism can occur by entry of air directly into pulmonary veins or systemic arteries. In Case 1, air was likely introduced into pulmonary veins at the time of lung biopsy or epinephrine injection. Air introduced into the systemic venous 


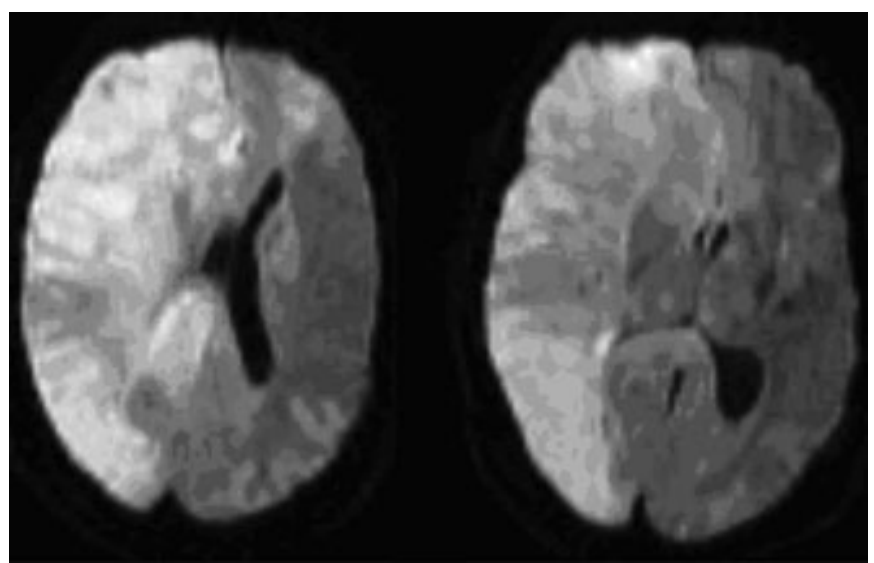

Figure 3: Case \#1 Axial diffusion-weighted MRI at 48 hours shows extensive restricted diffusion in the right cerebral hemisphere and in the left frontal and parietal lobes consistent with acute infarction. Significant mass effect has developed with right-to-left midline shift.

circulation may also pass through intracardiac (e.g. patent foramen ovale) or pulmonary shunts into the cerebral circulation ${ }^{1,2}$. When a large volume of air overwhelms the filtering capacity of the pulmonary vasculature, transpulmonary passage of air can lead to $\mathrm{CAE}^{1,2}$. Air introduced into systemic veins may also ascend retrograde to the cerebral veins. Due to the lower specific weight of air compared to blood, bubbles can ascend cranially with an upright body position. Obstruction of venous outflow due to air bubbles could lead to venous congestion, edema and infarct. Although the precise mechanism of CAE in Case 2 is unknown, the presence of air in the cavernous sinus is suggestive of venous air embolism, possibly due to an enterovenous shunt.

Arterial air emboli cause ischemic brain injury by direct occlusion of intracranial vessels, reducing cerebral perfusion to downstream tissues. They can also act as a foreign body, promoting inflammation and subsequent endothelial damage ${ }^{2}$. This causes leaky arterioles and cerebral edema, which leads to delayed ischemia even after the air emboli have dissipated. As air emboli are absorbed over hours, inflammation and brain edema cause an increase in intracranial pressure and subsequent reduction in cerebral perfusion ${ }^{2}$. A progressive decline in cerebral blood flow culminating in ischemic injury can occur some time after the onset of neurological symptoms, after air emboli are completely absorbed and can no longer be visualized on a NECT scan.

Despite a lack of randomized evidence demonstrating efficacy, hyperbaric oxygen therapy is considered the treatment of choice for CAE. The increased atmospheric pressure reduces the size of air bubbles and hyperoxia increases the oxygen carrying capacity of blood, facilitating delivery of oxygen to ischemic tissues. To improve cerebral perfusion and minimize infarction, immediate initiation of symptomatic therapies and hyperbaric oxygen treatment is recommended when a diagnosis of CAE is made. The prompt diagnosis of CAE is essential to achieve the best response possible to hyperbaric oxygen ${ }^{1,2}$.

Cerebral air embolism has largely remained a clinical diagnosis because of the low sensitivity of NECT to detect intra-

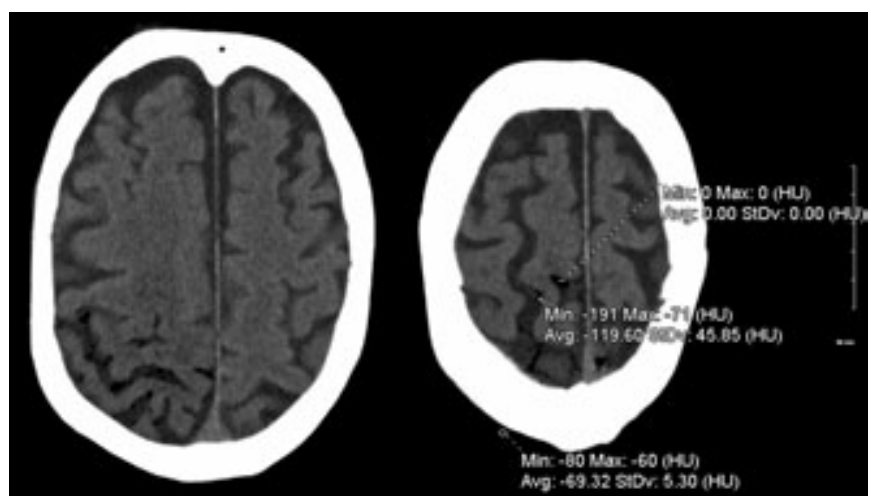

Figure 4: Case \#2 NECT. Multiple serpiginous foci of low attenuation are seen in the subarachnoid spaces of the right posterior parietal region (HU -119 or lower).

cranial air emboli ${ }^{4}$. Diffusion-weighted (DWI) MRI may improve diagnostic sensitivity and provide important prognostic information by demonstrating areas of ischemia and infarct. However, accessibility to MRI and lengthy scan times further delays diagnosis and therefore treatment time, limiting its effectiveness in the diagnosis of CAE.

Early CTP imaging in both patients described above demonstrated a large area of prominently increased TTP and

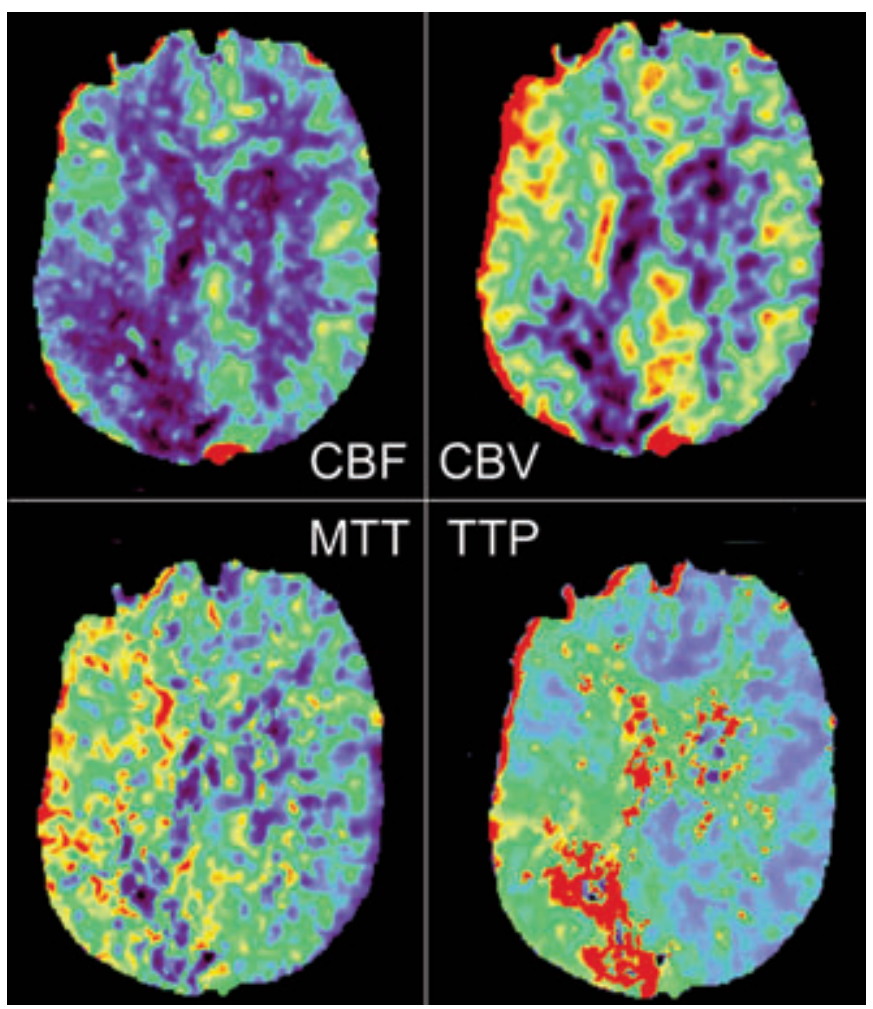

Figure 5: Case \#2 CT perfusion. Area of matched impaired CBF and $C B V$ shown in the right posterior parietal lobe is consistent with a core infarct. A larger area of prolonged MTT and TTP is evident in much of the right hemisphere representing the ischemic penumbra or tissue at risk. 
MTT, indicating that these parameters may be early, sensitive predictors of impending cerebral ischemia after CAE. In Case 1, $\mathrm{CBF}$ was only modestly decreased in the corresponding region with a normal CBV. Brain tissue with low CBV has been shown to be destined for infarction ${ }^{5}$. Thus, the lack of change in CBV in the first patient suggests the potential for salvageable brain tissue, provided perfusion can be restored promptly. In Case 2, a matched smaller area of impaired CBF and CBV was seen in the right parietal and occipital lobes, suggesting a core area of infarction 5 .

The two cases described above, illustrate that an imaging protocol consisting of a NECT of the head, CTA of the head and neck and brain CTP is ideal for the acute evaluation of CAE. The addition of CTA and CTP to NECT does not significantly increase imaging time; therefore it does not delay treatment, as is the case when transferring a patient from CT to MRI. This protocol can be performed on CT scanners that are widely available and do not require specialized equipment. Computed tomogram angiography provides important information on the patency of extra- and intra-cranial vessels and helps exclude other causes of neurological decline such as thromboembolic stroke. The CTP provides prognostic information regarding the severity of impending cerebral ischemia that may be useful for guiding management.
Non-enhanced CT alone is not completely reliable for detection of air emboli and use of MRI can unnecessarily delay diagnosis. These two cases illustrate that a $\mathrm{CT}$ imaging protocol utilizing a NECT of the head, CTA of the head and neck, and brain CTP was able to detect the presence of cerebral air emboli, rule out thrombus or stenosis in the extra- and intra-cranial arteries, and demonstrate areas of impaired cerebral perfusion and infarction. Thus, when used in conjunction, NECT, CTA and CTP may increase the diagnostic accuracy for CAE and provide important prognostic information.

\section{REFERENCES}

1. Muth CM, Shank ES. Gas embolism. N Engl J Med. 2000;342: 476-82.

2. van Hulst RA, Klein J, Lachmann B. Gas embolism: pathophysiology and treatment. Clin Physiol Funct Imaging. 2003;23: 237-46.

3. Menendez-Gonzalez M, Oliva-Nacarino P, Alvarez-Cofino A. Cerebral gas embolism caused by pleural fibrinolytic treatment. Stroke. 2007;38:2602-4.

4. Heckmann JG, Lang CJ, Kindler K, Huk W, Erbguth FJ, Neundörfer B. Neurologic manifestations of cerebral air embolism as a complication of central venous catheterization. Crit Care Med. 2000;28:1621-5.

5. Butcher K, Emery D. Acute stroke imaging. Part II. The ischemic penumbra. Can J Neurol Sci. 2010;37:17-27. 